\title{
Optic neuritis: chemokine receptor CXCR3 and its ligands
}

\section{T L Sørensen, H Roed, F Sellebjerg}

Br J Ophthalmol 2004;88:1146-1148. doi: 10.1136/bjo.2003.040980

Aim: To study the involvement of the chemokine receptor CXCR3 and its ligands (CXCL9/Mig, CXCL10/IP-10, CXCL11/ITAC) in optic neuritis (ON).

Methods: 30 patients with $\mathrm{ON}$ and 10 non-inflammatory neurological disease controls were included. The patients underwent a phlebotomy, lumbar puncture, and MRI scan. CXCR3 expression was studied on blood and cerebrospinal fluid (CSF) T cells by flow cytometry. CXCL9, CXCL10, and CXCL11 were measured in plasma and CSF by ELISA.

Results: CSF concentrations of CXCL10, but not of CXCL9 and $C X C L 11$, were significantly higher in $\mathrm{ON}$ patients than in controls. CSF concentrations of CXCL10 correlated with the CSF leucocyte count in ON patients, and CXCR3 expressing cells were significantly enriched in the CSF.

Conclusion: These data show that the CSF concentration of the CXCR3 ligand CXCL10 is selectively increased in CSF from $O N$ patients, and CXCR3 positive cells are recruited to the subarachnoid space.

\footnotetext{
O
} ptic neuritis (ON), frequently encountered by ophthalmologists, often represents the onset of multiple sclerosis (MS). ${ }^{1} \mathrm{MS}$ is an inflammatory disease of the central nervous system (CNS). ${ }^{2}$ The characteristics of the inflammatory lesion in the CNS are demyelination and axonal pathology with cellular infiltration characterised by the presence of phagocytic macrophages, T cells, and B cells. The inflammatory cell recruitment from the vascular bed to the perivascular space, and from here on to the CNS parenchyma, is the result of a multistep process, which is orchestrated in part by chemokines ${ }^{3}$; in other words, chemokines direct tissue invasion of specific inflammatory cells. The chemokine family is ever growing-now with more than 40 members. However, we and others have shown that the chemokine CXCL10 (also known as interferon inducible protein (IP)-10) and its receptor CXCR3 could be key players in the immunopathogenesis of MS, even though other receptor/ligand systems may also be of significance. ${ }^{5-10}$ CXCL10 is produced by astrocytes in the CNS during active inflammation and will attract CXCR3 expression cells into the site of inflammation. ${ }^{56}$ Since CXCR3 is expressed on activated Thl CD4+ and CD8+ T cells (and on B cells), the inflammatory composite around the CXCL10 producing astrocytes will be dominated by these cell types.

We have shown that CXCR3 expressing T cells and B cells are enriched in the cerebrospinal fluid (CSF) during acute relapses of MS, ${ }^{11}$ and in histopathology material CXCR3 + T cells in were primarily localised around CXCL10 positive astrocytes. The localised production of CXCL10 results in elevated CSF levels in patients compared to controls, and the CXCL10 levels correlate with the CSF leucocyte count. ${ }^{67}$ These findings provide strong cumulative evidence supporting a pivotal role for CXCR3 and its ligand CXCL10 in T cell recruitment to the brain in MS and, hence, that blocking this recruitment system might limit CNS inflammation in MS.
However, because most of the available data were obtained in established MS, and since the importance of commencing therapy very early in the disease course is increasingly recognised, we initiated a prospective study of patients with ON in order to investigate whether the CXCR3/CXCL10 axis has a similar role in patients presenting with a clinically isolated syndrome suggestive of MS. Since the CXCR3 receptor also has two other ligands (CXCL9, also known as Mig; and CXCL11, also known as ITAC), which have not been studied in this group of patients, we included CXCL9 and CXCL11 in our analysis. We correlated our data with measures related to the risk of developing MS (T2 weighted MRI lesions and IgG oligoclonal bands in CSF) or reflecting disease activity (CSF pleocytosis and number of gadolinium (Gd) enhancing lesions on MRI). We find that CXCL10 levels are elevated in the CSF of ON patients, and that CXCR3 expressing cells in the CSF correlate with the presence of oligoclonal bands in the CSF. This could suggest that CXCR3 blocking agents could be beneficial for all patients with ON.

\section{METHODS \\ Patients}

Thirty patients with ON were included in this study (20 female, 10 male, median age 36 years, range 21-55). An ophthalmologist examined all patients to rule out macula/ retinal disorders. An extensive medical history and paraclinical examination was performed on all patients to rule out any other potential cause of visual loss than ON (ischaemic episodes, tumours, etc). The clinical evaluation involved redwhite blood cell count, ANA, ANCA, ACE screening, visual evoked potentials, lumbar puncture, and magnetic resonance image (MRI). None of the patients had a previous history of neurological symptoms or clinical signs of spatial demyelination. As non-inflammatory neurological controls we included 10 patients (five female, five male, median age 48 , range 34 79). Eight had non-specific low back pain and two had spinal stenosis. None of the controls had oligoclonal bands in the CSF, and none had a CSF pleocytosis $>4$ cells $\times 10^{6} / 1$. Fifteen patients had oligoclonal bands present in the CSF, and 21 patients had abnormal MRI scans (more than two lesions). The median number of lesions was three (range 0-20). The median number of gadolinium enhancing lesions was one lesion (range 0-4).

\section{Preparation of cells, staining, flow cytometry, and ELISA}

CSF was obtained directly on ice, and centrifuged within 5 minutes of lumbar puncture. The supernatant was removed, snap frozen to $-80^{\circ} \mathrm{C}$, and was not thawed until ELISA was performed. Cells were resuspended in ice cold FACS phosphate buffered saline (PBS), incubated on ice with antibody and washed twice before analysis.

Abbreviations: CNS, central nervous system; CSF, cerebrospinal fluid; $M R I$, magnetic resonance mage; MS, multiple sclerosis; ON, optic neuritis; PBMC, peripheral blood mononuclear cells 
Table 1 Expression of CXCR3 on T cell subtypes in blood and CSF. Denoted in percentage, median and interquartile range (IQR)

\begin{tabular}{lllll}
\hline & $\begin{array}{l}\text { CXCR3 +CD4+ in blood } \\
\text { Median (IQR) }\end{array}$ & $\begin{array}{l}\text { CXCR3+ CD8+ in blood } \\
\text { Median (IQR) }\end{array}$ & $\begin{array}{l}\text { CXCR3+ CD4+ in CSF } \\
\text { Median }(\text { IQR) }\end{array}$ & $\begin{array}{l}\text { CXCR3+CD8+ in CSF } \\
\text { Median (CSF) }\end{array}$ \\
\hline ON & $33 \%(18,5)$ & $69 \%(29,5)$ & $82 \%(9,5)$ & $92 \%(13)$ \\
Controls & $27 \%(7)$ & $64 \%(20)$ & $89 \%(13)$ & $97 \%(7,5)$ \\
\hline
\end{tabular}

Blood mononuclear cells were obtained by density gradient centrifugation on Lymphoprep (Nycomed, Oslo, Norway), washed three times at $4^{\circ} \mathrm{C}$ in Hank's PBS with $1 \%$ human serum albumin and resuspended in ice cold FACS PBS $\left(10^{6}\right.$ cells/ml).

A volume of $100 \mu \mathrm{l}$ of peripheral blood mononuclear cells (PBMC) (100000 mononuclear cells) was incubated on ice with antibody for 30 minutes and washed twice in FACS PBS before analysis on a four colour FACSCalibur flow cytometer (Becton Dickinson), using CellQuest software (Becton Dickinson). Cells were gated according to forward light and side light scattering properties and CD4, CD8, or CD14 expression. Data are given as the percentage of CD4, CD8, or CD14 positive cells with immunofluorescence levels above the isotype control level expressed by $=1 \%$ of $\mathrm{CD} 3$ positive $\mathrm{T}$ cells. The following antibodies were used; FITC conjugated anti-CD4 (Clone SK3, Becton Dickinson Immunocytometry Systems, San Jose, CA, USA), PerCP conjugated anti-CD8 (Clone SK1, Becton Dickinson), APC conjugated anti-CD3 (Clone SK7, Becton Dickinson), PE conjugated anti-CXCR3 (Clone 1C6 Pharmingen). Isotype control antibody was PE conjugated mouse IgG1 (Clone MOPC-21, Becton Dickinson).

ELISA was performed on plasma and CSF for the chemokines CXCL9-11 using commercially available kits (R\&D Systems) according to the manufacturer's instructions. The intra-sample variability was less than $10 \%$.

\section{Statistics}

As results were not normally distributed (according to the Kolmogorow-Smirnoff test), statistical analysis was done with the non-parametric Mann-Whitney test for the analysis of unpaired data, and Spearman rank correlation analysis for analysing the relation between continuous variables. A 5\% significance level was employed.

\section{RESULTS}

\section{CXCR3 expressing $T$ cells are enriched into the CSF in} patients with ON

Data on CXCR3 expression are given in table 1. We found a significant enrichment of CXCR3 expressing CD4+ $(\mathrm{p}<0.001)$ and CD8 + T cells $(\mathrm{p}<0.01)$ into the CSF in patients with ON. This was also observed in neurological non-inflammatory controls. There was no difference in the percentage of CXCR3 positive CD4 or CD8 T cells in blood and CSF when values were compared in ON patients and non-inflammatory disease control subjects. The percentage of $\mathrm{CD} 4$ and CD8 T cells expressing CXCR3 did not correlate with the CSF leucocyte count or the number of active lesions on Gd enhanced MRI (data not shown). Neither the percentage of CXCR3 positive CD4 and CD8 $\mathrm{T}$ cells differ in ON patients with or without lesions on T2 weighted MRI or in patients with or with IgG oligoclonal bands in CSF (data not shown).

\section{CXCL10 levels are elevated in the CSF from patients}

We detected significantly higher levels of CXCL10 in the CSF from patients with ON compared to controls (table 2). Plasma concentrations of CXCL10 were low compared to CSF levels. There was no increase in the concentrations of CXCL9 or CXCL11 (table 2). The CXCL10 levels correlated with CSF leucocyte count in ON patients (Spearman's rho $=0.4739$, $\mathrm{p}<0.017$ ), and were slightly but not significantly higher in patients with IgG oligoclonal bands in CSF (data not shown, $\mathrm{p}<0.08$ ). CSF concentrations of CXCL10 were not higher in ON patients with an abnormal T2 weighted MRI, and did not correlate with the number of active lesion on Gd enhanced MRI. The levels of CXCLIO in the CSF did not correlate significantly with the percentage of CXCR3 expressing T cells in the blood or CSF.

\section{DISCUSSION}

Chemokines are essential in the process of inflammatory infiltrate formation in the CNS. A large number of studies have addressed the role of specific chemokines in the immunopathogenesis of MS, and in spite of more than 40 chemokines being known, the CXCR3/CXCL10 system appears to be of special importance. Because chemokine modulating drugs are emerging for the treatment of autoimmune disease, we set out to study the involvement of CXCL10 and CXCR3 in the development of ON-as part of a clinically isolated syndrome of demyelination-in order to provide the theoretical rationale for the initiation of treatment trials with CXCR3/CXCL10 modulating substances already at this stage of the disease.

There are strong data supporting a role for the CXCL10/ CXCR3 axis in MS immunopathogenesis. CXCR3 expressing cells are readily detected in MS lesions, they continue to accumulate throughout lesion development, CXCL10 is consistently elevated in CSF from MS patients, the CSF levels of CXCL10 correlate with inflammatory indicators and the number of cells in the CSF, and CXCL10 producing astrocytes are found in and around perivascular inflammatory cuffs. ${ }^{5-9} 11^{12}$ In the experimental model of MS-experimental allergic encephalomyelitis - the administration of anti-CXCL10 substances reduces $T$ cell recruitment into the CNS by approximately $50 \% .^{13}$

Table 2 CSF levels of chemokines (median and range)

\begin{tabular}{llll}
\hline & $\begin{array}{l}\text { CXCL9 (ng/ml) } \\
\text { Median (range) }\end{array}$ & $\begin{array}{l}\text { CXCL10 (pg/ml) } \\
\text { Median (range) }\end{array}$ & $\begin{array}{l}\text { CXCL11 (ng/ml) } \\
\text { Median (range) }\end{array}$ \\
\hline ON & $41(27-942)$ & $1146(226-3132)$ & $36(24-1875)$ \\
Controls & $52(29-1335)$ & $688(29-1335)$ & $42(25-123)$ \\
\hline
\end{tabular}


In this study we addressed whether this axis is altered in patients with ON without a history of MS. We find, in support of previous findings in patients with MS, that levels of CXCL10 are elevated in patients with ON compared to controls, and the levels correlate with the CSF leucocyte count-suggesting that CXCL10 actively recruit cells into the CNS during ON.

Since many of the patients have defective blood-brain barriers one could argue that the CXCL10 levels are the result of passive diffusion across a damaged blood-brain barrier. We have previously addressed this question in a series of MS patients where we found only minor amounts of CXCL10 in the periphery. ${ }^{7}$ This was confirmed in the present study where the amount of CXCL10 in CSF was about 10 times higher than in plasma. We previously found that CXCL9 concentrations were moderately elevated in CSF from patients with MS. ${ }^{6}$ This finding was not verified in the present study of patients with ON, and we found no increase in the CSF concentration of CXCL11 in ON. These findings are in agreement with histopathology studies showing limited (CXCL9) or absent (CXCL11) expression of these chemokines in MS lesions. ${ }^{5}$

Our findings are consistent with the notion that not only CXCR3 blocking but also CXCL10 blocking substances could prove effective in MS treatment since this seems to be the only CXCR3 ligand having a significant role in ON immunopathogenesis. Previous studies have failed in identifying a consistent relation between the expression of CXCR3 on $\mathrm{T}$ cells and disease activity in MRI studies. ${ }^{12}{ }^{14-16}$ In accordance with these studies, we found no relation between CXCL10, CXCR3 expression, and MRI disease activity, but we did find a trend towards higher CXCL10 levels in patients with IgG oligoclonal bands in CSF. This finding is in agreement with the results of a previous study where we found a correlation between the CSF concentration of CXCL10 and intrathecal IgG synthesis rates in patients with MS. These findings suggest that CXCL10 and CXCR3 are involved in the maintenance of chronic intrathecal inflammation in patients with ON and MS-and possibly the risk of future MS development.

Recent developments and the introduction of new diagnostic criteria-McDonald criteria-for MS have emphasised the importance of identifying and treating patients with ON correctly. ${ }^{17}$ The new McDonald criteria allow a definite MS diagnosis after a single clinically isolated syndrome-for example, ON, when dissemination in time and space is documented by paraclinical data. In addition, two recent studies have shown that treatment with interferon beta is efficacious in patients with clinically isolated syndromes and an abnormal MRI. ${ }^{18}{ }^{19}$ However, even though interferon beta is effective in limiting number and relapses and reducing disease progression more effective drugs are needed for the treatment of MS. The present study provides data supporting the rationale for clinical trials of substances interfering with the activity of CXCL10 and CXCR3 for the treatment of chronic $\mathrm{T}$ cell inflammation in patients with ON.
Authors' affiliations

T L Sørensen, H Roed, F Sellebjerg, The MS Clinic, Department of Neurology, University of Copenhagen, Glostrup Hospital, 2600

Glostrup, Denmark

Correspondence to: T L Sørensen, Department of Ophthalmology, University of Copenhagen, Herlev Hospital, 2720 Herlev, Denmark; torbenls@dadlnet.dk

Accepted for publication 29 February 2004

\section{REFERENCES}

1 Sorensen TL, Frederiksen JL, Bronnum-Hansen H, et al. Optic neuritis as onset manifestation of multiple sclerosis: a nationwide, long-term survey. Neurology 1999;53:473-8.

2 Sorensen TL, Ransohoff RM. Etiology and pathogenesis of multiple sclerosis. Sem Neurol 1998; 18:287-94.

3 Sellebjerg F, Sorensen TL. Chemokines and matrix metalloproteinase-9 in leukocyte recruitment to the central nervous system. Brain Res Bull 2003;61:347-55

4 Luster AD. Chemokines-chemotactic cytokines that mediate inflammation. N Engl J Med 1998;338:436-45

5 Balashov KE, Rottman JB, Weiner HL, et al. CCR5(+) and CXCR3(+) T cells are increased in multiple sclerosis and their ligands MIP-lalpha and IP-10 are expressed in demyelinating brain lesions. Proc Natl Acad Sci USA 1999;96:6873-8.

6 Sorensen TL, Tani M, Jensen J, et al. Expression of specific chemokines and chemokine receptors in the central nervous system of multiple sclerosis patients. J Clin Invest 1999;103:807-15.

7 Sorensen TL, Sellebjerg F, Jensen CV, et al. Chemokines CXCL10 and CCL2: differential involvement in intrathecal inflammation in multiple sclerosis. Eur J Neurol 2001;8:665-72.

8 Sorensen TL, Trebst C, Kivisakk P, et al. Multiple sclerosis: a study of CXCL10 and CXCR3 co-localization in the inflamed central nervous system. J Neuroimmunol 2002; 127:59-68

9 Franciotta D, Martino G, Zardini E, et al. Serum and CSF levels of MCP-1 and IP-10 in multiple sclerosis patients with acute and stable disease and undergoing immunomodulatory therapies. J Neuroimmunol 2001;115:192-8

10 Teleshova N, Pashenkov M, Huang YM, et al. Multiple sclerosis and optic neuritis: CCR5 and CXCR3 expressing T cells are augmented in blood and cerebrospinal fluid. J Neurol 2002;249:723-9.

11 Sorensen TL, Roed H, Sellebjerg F. Chemokine receptor expression on B cells and effect of interferon-beta in multiple sclerosis. J Neuroimmunol 2002; 122:125-31.

12 Sindern E, Patzold T, Ossege LM, et al. Expression of chemokine receptor CXCR3 on cerebrospinal fluid T-cells is related to active MRI lesion appearance in patients with relapsing-remitting multiple sclerosis. J Neuroimmunol 2002;131:186-90.

13 Fife BT, Kennedy KJ, Paniagua MC, et al. CXCL10 (IFN-gamma-inducible protein-10) control of encephalitogenic CD4+ T cell accumulation in the central nervous system during experimental autoimmune encephalomyelitis. $J$ Immunol 2001; 166:7617-24.

14 Wang HY, Matsui M, Araya S, et al. Chemokine receptors associated with immunity within and outside the central nervous system in early relapsingremitting multiple sclerosis. J Neuroimmunol 2002;133:184-92.

15 Eikelenboom MJ, Killestein J, Izeboud T, et al. Chemokine receptor expression on T cells is related to new lesion development in multiple sclerosis. J Neuroimmunol 2002;133:225-32.

16 Putheti P, Morris M, Stawiarz L, et al. Multiple sclerosis: a study of chemokine receptors and regulatory T cells in relation to MRI variables. Eur J Neurol 2003; 10:529-35.

17 McDonald WI, Compston A, Edan G, et al. Recommended diagnostic criteria for multiple sclerosis: guidelines from the international panel on the diagnosis of multiple sclerosis. Ann Neurol 2001;50:121-7.

18 PRISMS. Randomised double-blind placebo-controlled study of interferon beta-la in relapsing/remitting multiple sclerosis. PRISMS (Prevention of Relapses and Disability by Interferon beta-1 a Subcutaneously in Multiple Sclerosis) Study Group. Lancet 1998;352:1498-504.

19 Comi G, Filippi $M$, Barkhof $F$, et al. Effect of early interferon treatment on conversion to definite multiple sclerosis: a randomised study. Lancet $2001 ; 357: 1576-82$. 